\title{
Quenching Effect of Blood on Fluorescent Glucose Analogue NBDG
}

\author{
Hana Hayasaki ${ }^{1}$, Masahito Watanabe ${ }^{1}$, Kiyoto Kanbara ${ }^{1}$, Nobuo $\mathbf{J o}^{2}$, \\ Masahiro Takafuchi ${ }^{3}$, Yutaka Watanabe ${ }^{1}$ and Masahisa Shimada ${ }^{1}$ \\ ${ }^{1}$ Departments of Anatomy, ${ }^{2}$ Dermatology and ${ }^{3}$ Central Research Laboratory, Osaka Medical College, 2-7, \\ Daigakumachi, Takatsuki City, Osaka 569
}

Received for publication January 26, 1996 and in revised form July 17, 1996

\begin{abstract}
A fluorescent glucose analogue, 6-deoxy- $\mathrm{N}$ (7-nitrobenz-2-oxa-1,3-diazol-4-yl)-aminoglucose (NBDG) has been used for studies of glucose transport. The fluorescence from NBDG was observed with a confocal laser scanning microscope (CLSM). The fluorescence in the blood vessels, however, could not be observed even though substantial amount of NBDG was present in the blood. We investigated the causative fac-
\end{abstract}

tors responsible for this non-fluorescence. It was found that NBDG made an uncertain complex with hemoglobin which decreased the fluorescence intensity of NBDG. However, it appeared that the principal causative factor was absorbance of excitation and emission wave lengths by hemoglobin which resulted in a significant decrease of fluorescence intensity from NBDG in the blood vessels.

Key words: NBDG, Fluorescence, Quenching, Hemoglobin

\section{Introduction}

In the previous work, we studied glucose transport in brain tissue by using 6-deoxy-N- (7-nitrobenz-2-oxa-1, 3diazol-4-yl)-aminoglucose (NBDG) and confocal laser scanning microscope (CLSM) [9]. The fluorescent labeling compound, NBDG, has an excitation and emission maximum of $480 \mathrm{~nm}$ and $550 \mathrm{~nm}$, respectively. Therefore when histological sections were excited with an Argon laser at $488 \mathrm{~nm}$ of a CLSM, we could observe the fluorescence of wavelength $550 \mathrm{~nm}$ in the regions where NBDG was present. In the previous work, however, it was found that even if the NBDG was introduced into the mouse blood vessel directly and it was killed 1 min later, no fluorescence was observed in the blood of the brain micro vessels under the low power magnification [9]. This phenomenon seems to result from the quenching of fluorescence by certain blood components. To confirm this phenomenon, NBDG was injected into mouse tail vein and the liver was removed at $5 \mathrm{~min}$. Because NBDG is water soluble, we prepared freeze-dried sections, and then used the CLSM to observe fluorescence. The fluorescence from NBDG was seen in the liver parenchyma, while no fluorescence was observed in the blood vessels, including

Correspondence to: Professor Masahisa Shimada, Department of Anatomy, Osaka Medical College, 2-7, Daigakumachi, Takatsuki City, Osaka 569, Japan. sinusoids (Fig. 1). Although it is known that blood has a quenching effect on fluorophores [1-3, 5, 11], their mechanisms are still open to question. The present work was designed to investigate the causative factors responsible for the absence of fluorescence in the blood and revealed that a main factor is absorbance of excitation and emission lights by hemoglobin. To ascertain this, we investigated tetracycline as well. The procedure on sections of tetracycline was similar to that of NBDG, and sections were observed with a conventional fluorescence microscope with a $\mathrm{Hg}$ lamp.

\section{Materials and Methods}

\section{Reagents}

NBDG was purchased from the Molecular Probes Inc. (Eugene, Oregon). Hemoglobin (bovine), albumin (bovine serum) and tetracycline were purchased from Sigma Chemical Corporation (St. Louis, Missouri). Trichloroacetic acid (TCA) was purchased from Wako Pure Chemical Industries Ltd. (Osaka, Japan). The PBS consisted of $0.15 \mathrm{M} \mathrm{NaCl}$ and $0.01 \mathrm{M}$ sodium phosphate buffer, and the $\mathrm{pH}$ was 7.4.

\section{Preparation of histological sections}

Albino mice of the ICR strain (Clea Japan. Co., Osaka, Japan), weighing approximately $30 \mathrm{~g}$, were used. At 5 min after intravenous injection of $25.3 \mu \mathrm{g}$ of NBDG 
or tetracycline dissolved in physiological saline, the liver was removed and quickly frozen in Freon, and then cooled again in liquid nitrogen according to the method of Shimada et al. [8]. Sections $25 \mu \mathrm{m}$ thick, were cut in a cryostat at $-15^{\circ} \mathrm{C}$. After freeze-drying on cold gelatincoated slides, the sections were immersed in xylene, and mounted in immersion oil. In the case of sections of NBDG, we used a Carl Zeiss confocal laser scanning microscope (LSM-10, Oberkochen, Germany) with an Argon laser at $488 \mathrm{~nm}$. The histological section for tetracycline was observed with a fluorescence microscope with a Hg lamp.

\section{Quantitative analysis of NBDG in the blood}

At $45 \mathrm{sec}$ after intravenous injection of $25.3 \mu \mathrm{g}$ of NBDG, the blood was collected from the heart, and stocked in heparinized test tubes. The blood was centrifuged at $3000 \mathrm{rpm}$ for $10 \mathrm{~min}$, and divided into whole blood, erythrocytes, and serum. The erythrocytes were centrifuged at $3000 \mathrm{rpm}$ after adding cold 50\% TCA. The concentration of NBDG was measured with a fluorescence spectrophotometer (M-850, Hitachi Co., Tokyo, Japan) with the quartz cuvettes having $1 \mathrm{~cm}$ path-length and $3 \mathrm{ml}$ capacity. Calibration curves made from the measurements of supernatants of the blood components were used to determine the NBDG concentrations. The supernatants contained known quantities of NBDG. In addition, to investigate which blood components influence the quenching effect, whole blood, erythrocytes and serum were smeared on gelatinized glass slides, dried, mounted with squalane oil, and observed with a CLSM.

\section{Effects of blood components on the fluorescence intensity}

To investigate the effects of blood components on the fluorescence intensity, we prepared the following solutions. Solution A was a phosphate buffer ( $\mathrm{pH}$ 7.4). Solution B contained the blood dissolved in phosphate buffer. Solution $C$ had erythrocytes dissolved in phosphate buffer. Solution D contained hemolyzed erythrocytes in phosphate buffer. Solution $\mathrm{E}$ had the supernatant of hemolyzed erythrocytes and 50\% cold TCA, centrifuged at $3000 \mathrm{rpm}$ for $10 \mathrm{~min}$. Solutions B, C and D contained $0.025 \mathrm{~g} / \mathrm{dl}$ hemoglobin each. After adding various concentrations of NBDG to each $6 \mathrm{ml}$ of solution, the fluorescence intensities were measured with a fluorescence spectrophotometer with $390 \mathrm{~nm}$ and $488 \mathrm{~nm}$ for excitation and emission.

\section{Absorption spectra of hemoglobin}

To see the absorption spectra of hemoglobin, $0.5 \mathrm{~g} / \mathrm{dl}$ hemoglobin and $0.007 \mathrm{~g} / \mathrm{dl}$ albumin were dissolved in PBS, respectively. For measuring the absorption band of hemoglobin, a spectrophotometer was used to scan from 420 to $700 \mathrm{~nm}$.

\section{Transmissivity of the hemoglobin solution}

To measure the transmissivity of the hemoglobin solu- tion, various concentrations of hemoglobin in $2 \mathrm{ml}$ of PBS were prepared. Transmissivity was measured at 390 and $520 \mathrm{~nm}$ for tetracycline, 488 and $550 \mathrm{~nm}$ for NBDG with spectrophotometer. Transmissivity showed hemoglobin absorbs light of excitation and emission.

\section{Influence of hemoglobin on fluorescence intensities of NBDG and tetracycline}

To observe the influence of hemoglobin on the fluorescence intensity of NBDG and tetracycline, the following samples were prepared. Solutions of $6 \mathrm{ml}$ of PBS containing $0 \sim 250 \mu \mathrm{g} / \mathrm{dl}$ hemoglobin were prepared. Into some of these solutions $1 \mu \mathrm{g}$ of NBDG was added and into others $2 \mu \mathrm{g}$ of tetracycline were added. Fluorescence intensities of each sample were measured with a fluorescence spectrophotometer with $390 \mathrm{~nm}$ and $488 \mathrm{~nm}$ for excitation and emission of tetracycline respectively, and $488 \mathrm{~nm}$ and $550 \mathrm{~nm}$ for those of NBDG. The width of the excitation slit was $2 \mathrm{~nm}$.

\section{Binding effects of $N B D G$ with hemoglobin}

To examine whether NBDG form complex with hemoglobin, NBDG was incubated with hemoglobin. The solution of $0.5 \mathrm{ml}$ of PBS containing $4.5 \mathrm{~g} / \mathrm{dl}$ of hemoglobin and $1 \mu \mathrm{g}$ of NBDG was prepared. As the control, $0.5 \mathrm{ml}$ of PBS containing $1 \mu \mathrm{g}$ of NBDG was prepared. Both solutions were incubated at $37^{\circ} \mathrm{C}$ for 0 to $60 \mathrm{~min}$, and then $0.5 \mathrm{ml}$ of $50 \%$ TCA was added to each sample after its designated incubation time. After being centrifuged at $3000 \mathrm{rpm}$, the supernatants were measured with fluorescence spectrophotometer.

To know whether fluorescence intensity of the NBDG-Hb complex differs from that of NBDG and hemoglobin conjugate, the following experiments were made. Solutions of $0.5 \mathrm{ml}$ PBS containing hemoglobin of concentration $4.5 \mathrm{~g} / \mathrm{dl}$ and $0.2 \mathrm{ml}$ PBS containing $1 \mu \mathrm{g}$ NBDG were prepared. These solutions were incubated at $37^{\circ} \mathrm{C}$ for 0 to $60 \mathrm{~min}$. However, in one group of the experiment, two of the solutions were mixed before incubation. In the other group, two solutions were incubated separately and mixed immediately prior to being measured with the fluorescence. The solutions were diluted with $5.5 \mathrm{ml}$ of PBS before measurement of fluorescence intensity with the spectrophotometer.

\section{Results}

At 5 min after intravenous injection of NBDG, fluorescence intensities were highly concentrated in the portal vein of the liver parenchyma, while no fluorescence was observed in the blood vessels including interlobular portal veins, sinusoids and central veins (Fig. 1). The same image was observed in the liver at $5 \mathrm{~min}$ after intravenous injection of tetracycline (Fig. 2). Quantitative analysis of NBDG in the whole blood, erythrocytes and the serum by the spectrophotometer showed that the concentrations were $0.10 \mu \mathrm{g} / \mathrm{ml}$ in the blood, $0.68 \mu \mathrm{g} / \mathrm{ml}$ in the 


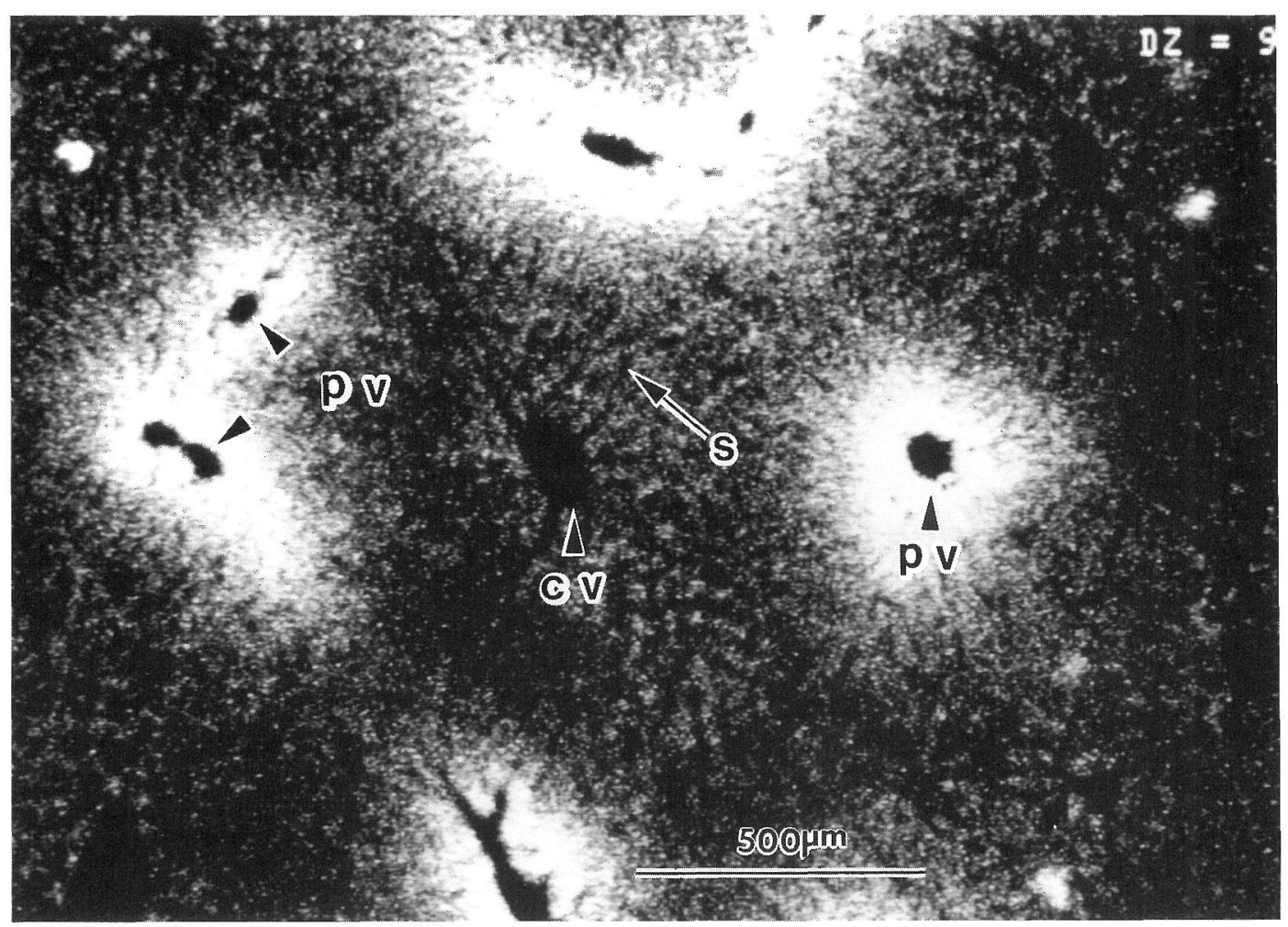

Fig. 1 Confocal image of fluorescence of a mouse liver $5 \mathrm{~min}$ after NBDG injection. No fluorescence was observed in the portal veins (PV), the sinusoids (S) and the central veins (CV). Excitation: $488 \mathrm{~nm}$ Argon Laser. $\times 64$.

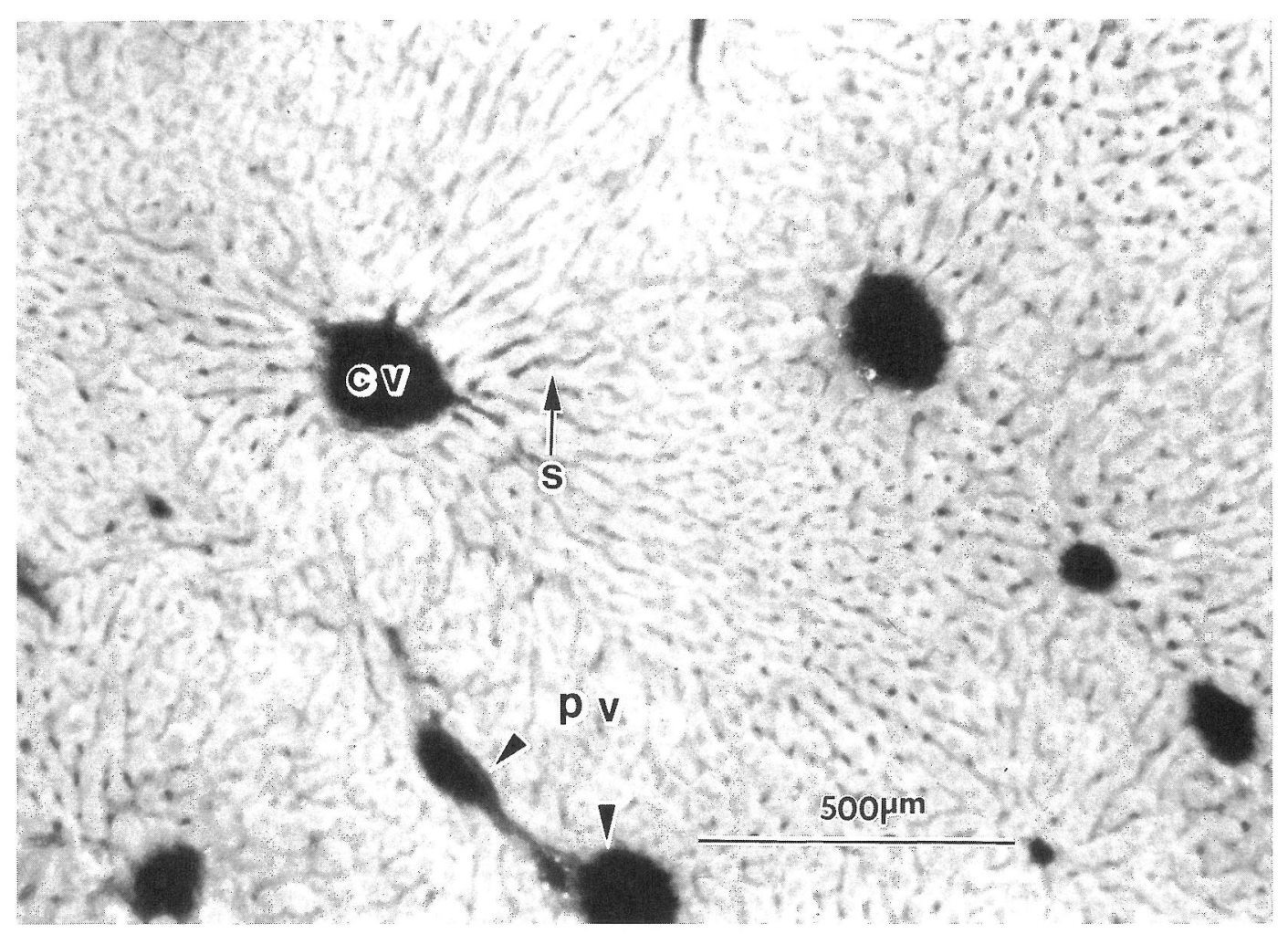

Fig. 2. Fluorescence image of a mouse liver $5 \mathrm{~min}$ after tetracycline injection observed with conventional fluorescent microscope with a $\mathrm{Hg}$ lamp. Note no fluorescence in the portal veins (PV), the sinusoids $(\mathrm{S})$ and the central veins $(\mathrm{CV}) . \times 70$. 
erythrocytes and $1.50 \mu \mathrm{g} / \mathrm{ml}$ in the serum, at $5 \mathrm{~min}$ after injection of $25.3 \mu \mathrm{g}$ NBDG. During observation of the smear of blood with CLSM, however, no fluorescence was observed except in the serum.

\section{The quenching effect of hemoglobin (Fig. 3)}

Fluorescence intensity increases with the concentrations of NBDG in the solution A. However, the fluorescence intensity of the solutions containing hemoglobin (solutions B, C and D) were significantly lower than that of solutions without hemoglobin (solutions $\mathrm{A}$ and $\mathrm{E})$.

\section{Absorption spectra of hemoglobin}

Figure 4 shows an absorption spectra of hemoglobin and albumin. The hemoglobin has an absorption peak at $500 \mathrm{~nm}$, while albumin does not show any absorption peak between 420 and $700 \mathrm{~nm}$. As illustrated in Figure 4, hemoglobin shows high spectra absorption at $488 \mathrm{~nm}$ and $550 \mathrm{~nm}$ as compared to that of albumin. Excitation and emission wavelengths of NBDG were 488 and $550 \mathrm{~nm}$, respectively.

\section{Transmissivity of hemoglobin solutions (Figs. 5 and 6)}

The influence of hemoglobin on transmissivity was observed by changing the concentration of hemoglobin. The transmissivity of excitation of tetracycline at $390 \mathrm{~nm}$ and NBDG at $488 \mathrm{~nm}$ was decreasing as the hemoglobin concentrations increased. The transmissivity of emission of tetracycline at $520 \mathrm{~nm}$ and NBDG at $550 \mathrm{~nm}$ also appeared to be decreasing with the hemoglobin concentra-

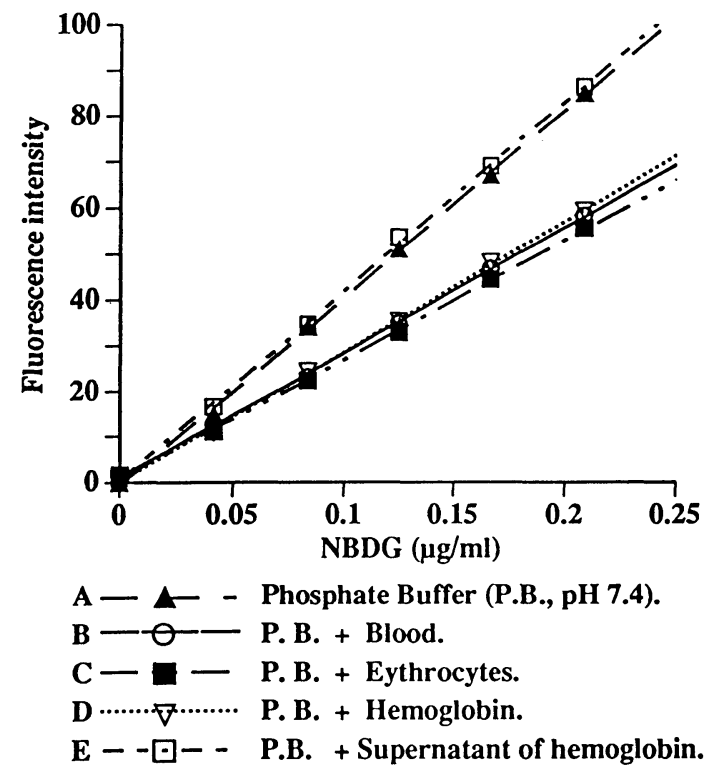

Fig. 3. Quenching effect of blood components on the fluorescence intensity. Solution A and E did not contain hemoglobin. Solution $\mathrm{B}, \mathrm{C}$ and $\mathrm{D}$ contained $0.025 \mathrm{~g} / \mathrm{dl}$ hemoglobin each. Note that the solutions containing hemoglobin decreased the fluorescent intensity of NBDG. tions. The hemoglobin had a significantly larger influence on tetracycline at the excitation and the emission wavelength than on NBDG. From this data and the Lambert-Beer Law, fluorescence intensity with the various concentrations of hemoglobin is calculated

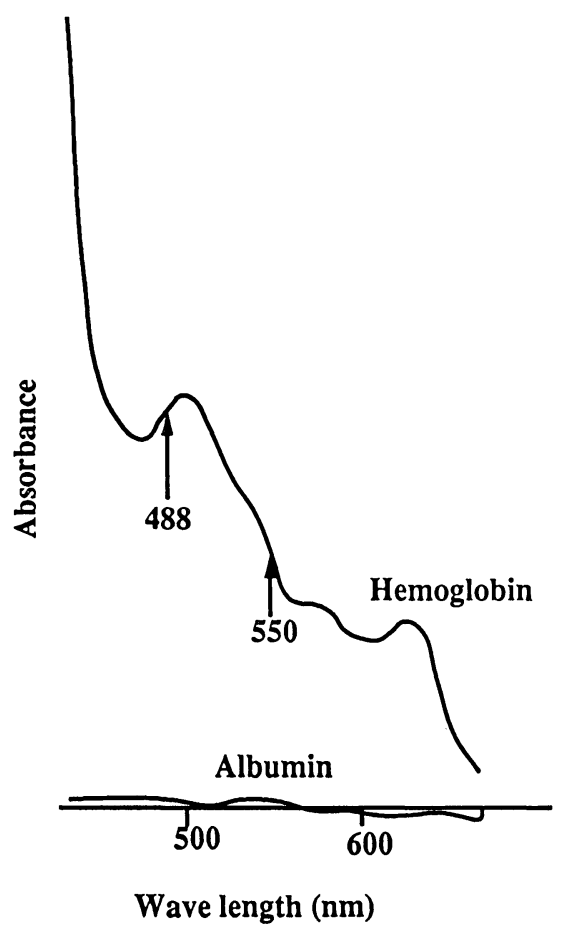

Fig. 4. Absorption spectra of hemoglobin and albumin. The hemoglobin solution contained $0.5 \mathrm{~g} / \mathrm{dl}$ hemoglobin and the albmin solution contained $0.007 \mathrm{~g} / \mathrm{dl}$. Arrows point to the NBDG excitation and emission (488 and $550 \mathrm{~nm}$ ). The hemoglobin has a high absorption at 488 and $550 \mathrm{~nm}$, which albumin does not.

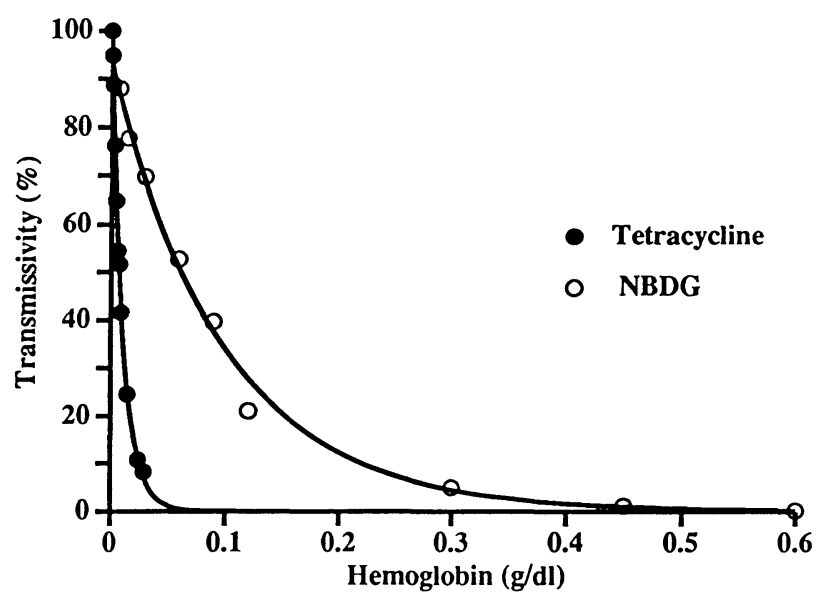

Fig. 5. Alteration of transmissivity of NBDG and tetracycline by hemoglobin at excitation 488 and $390 \mathrm{~nm}$ respectively. The hemoglobin values range from 0 to $0.6 \mathrm{~g} / \mathrm{dl}$ in PBS containing NBDG or tetracycline. Transmissivity is indicated as the relative decrease of fluorescence intensity by hemoglobin. 


$$
\begin{aligned}
& \log \left(\mathrm{I}_{0} / \mathrm{I}\right)=-\mathrm{E} \times 10^{3} \times \mathrm{d} \times \mathrm{C} \\
& \log \left(\mathrm{F}_{0} / \mathrm{F}\right)=-\mathrm{E} \times 10^{3} \times \mathrm{d} \times \mathrm{C}
\end{aligned}
$$

where $I_{0}$ and $I$ are the light intensities of the excitation beams of tetracycline $(390 \mathrm{~nm})$ or NBDG $(488 \mathrm{~nm})$, and $\mathrm{F}_{0}$ and $\mathrm{F}$ represent those of emission beams of tetracycline $(520 \mathrm{~nm})$ or NBDG $(550 \mathrm{~nm})$. $E(55.8,6.54,6.54,4.98)$ are the molar extinction coefficients of hemoglobin at 390 , $488,520,550 \mathrm{~nm}$, respectively. $\mathrm{C}$ is the molarity of hemoglobin, and $d$ is light length. Figure 7 shows that the fluorescence intensities decreased as the hemoglobin concentrations increased. Therefore, increasing concentrations of hemoglobin have greater influence on fluorescence intensities of the fluorescent materials.

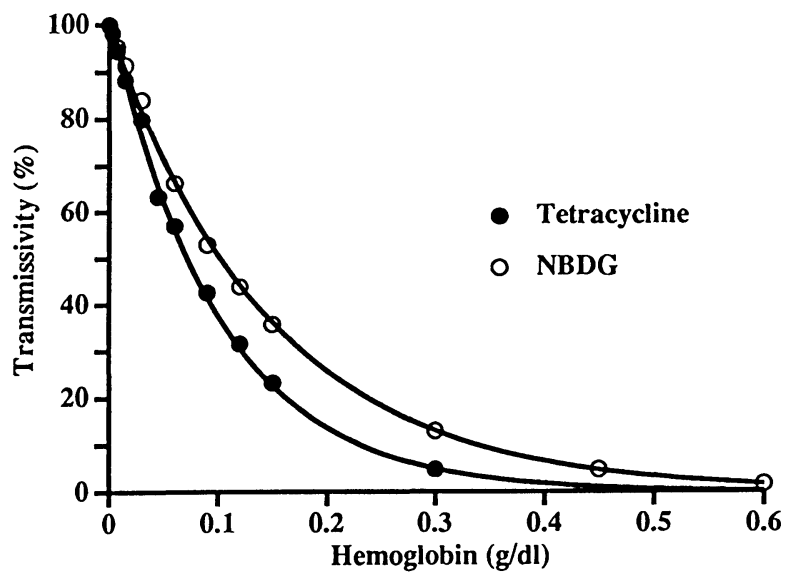

Fig. 6. Alteration of transmissivity of NBDG and tetracycline by hemoglobin at emission 520 and $550 \mathrm{~nm}$ respectively. The hemoglobin values range from 0 to $0.6 \mathrm{~g} / \mathrm{dl}$ in PBS containing NBDG or tetracycline. Transmissivity is indicated as the relative decrease of fluorescence intensity by hemoglobin.

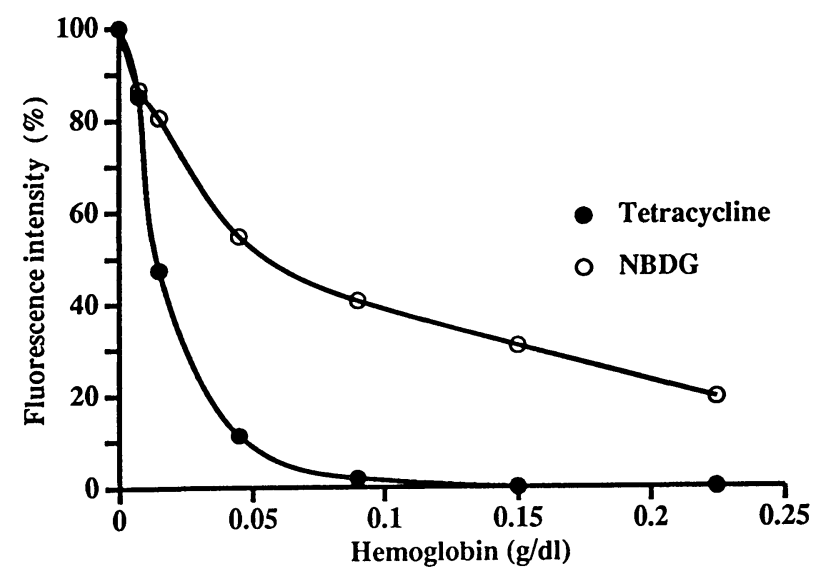

Fig. 7. The relationship of fluorescence intensity of NBDG or tetracycline and concentration of hemoglobin. The hemoglobin valies raging from 0 to $0.225 \mathrm{~g} / \mathrm{dl}$ in PBS containing NBDG or tetracycline.

\section{Binding effects of NBDG with hemoglobin}

The formation of the NBDG-hemoglobin complex increased as incubation time increased up to $60 \mathrm{~min}$ (Fig. 8). Furthermore, it is clear that the complex has quenching effect (Fig. 9).

\section{Discussion}

At 5 min after intravenous injection of NBDG, intensive fluorescence was observed in the liver, while not in the blood. To make sure of the non fluorescent effect in the blood, the blood was smeared on glass slides after intravenous injection of NBDG and observed by CLSM together with quantitative analysis of the NBDG contents. As a result, it was found that intensive fluorescence of NBDG was observed in the serum, while not in the

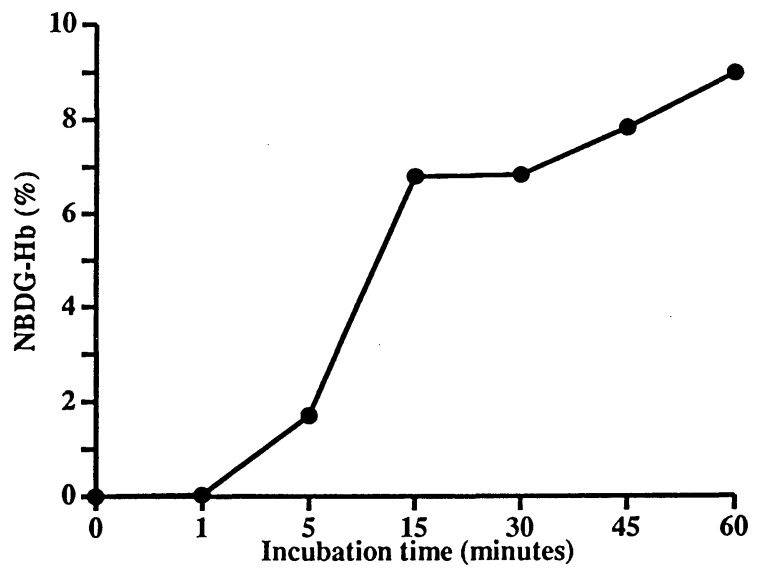

Fig. 8. Binding of NBDG with hemoglobin. The solution of $0.5 \mathrm{ml}$ of PBS is containing $4.5 \mathrm{~g} / \mathrm{dl}$ of hemoglobin and $1 \mu \mathrm{g}$ of NBDG was incubation at $37^{\circ} \mathrm{C}$.

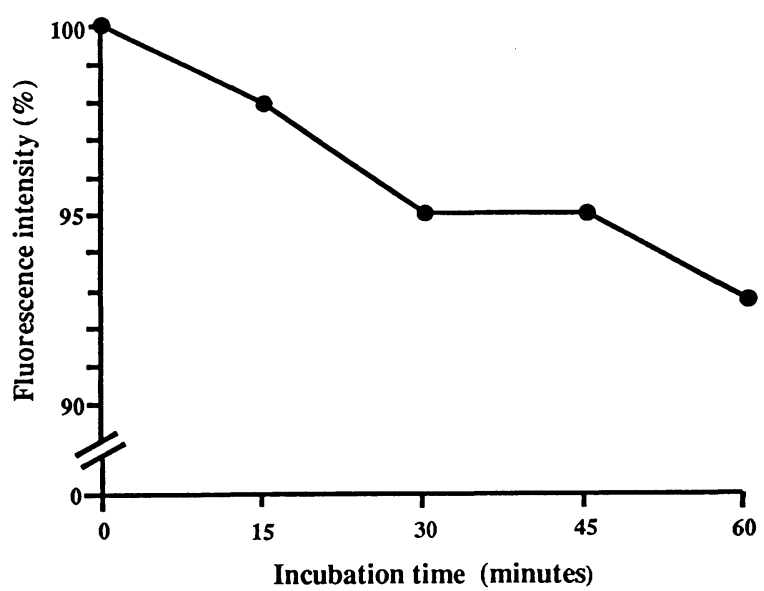

Fig. 9. Change of fluorescence intensity of NBDG incubated with hemoglobin. One solution of $0.5 \mathrm{ml}$ of PBS is containing $4.5 \mathrm{~g} / \mathrm{dl}$ hemoglobin, the other solution of $0.2 \mathrm{ml}$ of PBS is containing $1 \mu \mathrm{g}$ of NBDG. Fluorescence intensity is indicated as the relative decrease of the intensity against NBDG and hemoglobin conjugate. 
whole blood and erythrocytes even though all fractions contained substantial amount of NBDG.

As the next step, we studied the effects of blood elements on the fluorescence intensity of NBDG. Fluorescence intensities of the solutions containing hemoglobin were significantly lower than those solutions without hemoglobin. This implies that hemoglobin may strongly quench the fluorescence. Generally, the fluorescence is influenced by several factors such as temperature, $\mathrm{pH}$, kind of solutions, and existence of the third material [4]. Here we investigated hemoglobin as the third material for NBDG. Hemoglobin has strong absorbance at the excitation and emission wavelength of both NBDG and tetracycline. Thus fluorescence intensities of NBDG and tetracycline are greatly influenced by hemoglobin. We then investigated transmissivity in solutions of hemoglobin at the excitation and emission wavelength of NBDG and tetracycline. Consequently, it was found that the light of excitation of tetracycline at $390 \mathrm{~nm}$ and NBDG at $488 \mathrm{~nm}$ decreased when the hemoglobin concentrations were increased. The transmissivity of $390 \mathrm{~nm}$ was lower than that of $488 \mathrm{~nm}$, as hemoglobin has a maximum at $415 \mathrm{~nm}$ which is closer to $390 \mathrm{~nm}$. The light of emission of tetracycline at $520 \mathrm{~nm}$ and NBDG at $550 \mathrm{~nm}$ also appeared to decrease by increasing the NBDG concentration. The transmissivity at emission wavelength of tetracycline was also significantly lower than at that of NBDG. So fluorescence intensity of tetracycline may be more influenced by hemoglobin than that of NBDG. The data was converted to absorbance values, and analyzed using the Lambert-Beer Law. We calculated the decreasing fluorescence intensity in various concentrations of hemoglobin. The attenuation of the exciting and emitting light within the section could be given by formulas from equation I or II. $\mathrm{I}_{0}$ (the light intensities of the excitation beams) was given to the sections. When $\mathrm{I}_{0}$ traveled $\mathrm{d} \mathrm{cm}$ in approaching the fluorescent substance, the light intensities of the excitation beams might decrease from $\mathrm{I}_{0}$ to $\mathrm{I}$. The fluorescent substance was excited by I and might radiate the fluorescence $\left(F_{0}\right)$. When the fluorescence intensities of the emission beams $\left(\mathrm{F}_{0}\right)$ traveled $\mathrm{d}^{\prime} \mathrm{cm}$, it may decrease from $\mathrm{F}_{0}$ to $\mathrm{F}$. We related the absorbance of exciting and emitting lights with the decrease of fluorescence intensities by the hemoglobin. This indicated that if both hemoglobin and NBDG are distributed uniformly within the section, fluorescence from NBDG is decreased by hemoglobin [5]. To study the relationship of fluorescence intensity and concentration of hemoglobin, we measured the fluorescence intensities of fluorescent materials in the presence of varying concentrations of hemoglobin. Figure 7 shows the relationship between hemoglobin concentration and fluorescence intensity. The fluorescence intensity of tetracycline was significantly lower than that of NBDG, this may be caused by greater absorption of exciting and emitting lights by the hemoglobin. However, hemoglobin may also markedly quench the fluorescence of NBDG.
Fluorescence quenching refers to any process which decreases the fluorescence intensity of a substance. Various processes may result in quenching. These include energy transfer, complex formation, and collisional quenching [4]. In the present work it was found that quenching could occur because of the optical properties of the sample. This process may belong to diffusion of energy. Static quenching may arise from the formation of a dark complex between fluorophore and the quenching agent [14]. For instance, hemoglobin is an efficient quenching agent for several aromatic fluorescent dyes, and the quenching has been determined to be the result of a dyehemoglobin complex [11]. Therefore, we examined the possible complex formation between the fluorophore and quencher, this is called static quenching. Mouse hemolysate contains several minor components designated as glycosylated hemoglobin. NBDG is a fluorescent glucose analogue because aminoglucose is labeled with NBD at the sixth carbon position. So, it is possible that NBDG might bind with $\mathrm{Hb}$, to form NBDG-Hb complex $[6,7,10]$.

$$
\mathrm{NBDG}+\mathrm{Hb} \leftrightarrows \mathrm{NBDG}-\mathrm{Hb}
$$

If the purified hemoglobin is incubated with NBDG, NBDG would form a covalent bond with hemoglobin through a process similar to the nonenzymatic glycosylation of hemoglobin as shown by Speizer and colleagues [7]. In the present study, the complex formation (Fig. 8) and the complex's quenching effect (Fig. 9) have been ascertained. These results indicate that NBDG-Hb might slightly quench NBDG, but the complex influences fluorescence intensity very little in $5 \mathrm{~min}$. In summary, hemoglobin had a decreasing influence on the fluorescence intensity of NBDG. The mechanism is that hemoglobin absorbed the light of excitation and emission wave length, and this absorbance was different for the various fluorescent materials. Hemoglobin forms an uncertain complex with NBDG, but the fluorescence intensity of the complex was at least as low as that of NBDG. Although hemoglobin had two kinds of quenching, the significant quenching effect on NBDG is absorbance of light excitation and emission. Therefore, the observation of no fluorescence in the blood vessels by the CLSM may depend on the factor. Apart from the hemoglobin, there are many proteins containing heme proteins such as cytochrome $C$ and peroxidases. These compound may also certain quenching effects on the fluorescence of NBDG. However, under the assumption that these quenching effects are even and very low in most parenchymal tissues as compared with blood, NBDG can be used to visualize glucose transport in the tissues.

\section{References}

1. Gonzalez, J. J., Jacquotte, H. and Cayre, I.: Fluorescence quenching studies on binding fluoreno-9-spiro-oxazolidinedione to human serum albumin. Chem. Biol. Interact. 84; 221-228, 
1992.

2. Horiuchi, K. and Asai, H.: A reaction cell for simultaneous measurements of fluorescence and absorption in a hemoglobin solution at various oxygen pressures. Biochem. Biophys. Res. Commun. 97; 811-818, 1980.

3. Kuwajima, T. and Asai, H.: Synthesis of fluorescent organic phosphates and their equilibrium binding to bovine oxyhemoglobin. Biochemistry 14; 492-497, 1975.

4. Lakowicz, R. J.: Principles of fluorescence spectroscopy. Plenum Press, New York, 1983, pp. 257-301.

5. Lew, V. L., Etzion, Z., Bookchin, R. M., DaCosta, R., Väänänen, H., Sassaroli, M. and Eisinger, J.: The distribution of intracellular calcium chelator (fura-2) in a population of intact human red cells. Biochim. Biophys. Acta 1148; 152-156, 1993.

6. Macias, P., Pinto, M. C. and Merino, C. G.: Hemin and hemeprotein bleaching during linoleic acid oxidation by lipoxygenases. Biochim. Biophys. Acta 1082; 310-318, 1991.
7. McDonald, M. J., Shapiro, R., Bleichman, M., Solway, J. and Bunn, H. F.: Glycosylated minor components of human adult hemoglobin. J. Biol. Chem. 253; 2327-2332, 1978.

8. Shimada, M., Shimono, R. and Ozaki, H. S.: Freeze-mount microautoradiographic study in the mouse hippocampus after intravenous injection of tritiated 2-deoxyglucose and glucose. Neuroscience 31; 347-354, 1989.

9. Shimada, M., Kawamoto, S., Hirose, Y., Nakanishi, M., Watanabe, H. and Watanabe, M.: Regional difference in glucose transport in the mouse hippocampus. Histochem. J. 26; 207-212, 1994.

10. Speizer, L., Haugland, R. and Kutchai, H.: Asymmetric transport of a fluorescent glucose analogue by human erythrocytes. Biochim. Biophys. Acta 815; 75-84, 1985.

11. Wilson, D. H., Bogacz, J. P., Forsythe, C. M., Turk, P. J., Lane, T. L., Gates, R. C. and Brandt, D. R.: Fully automated assay of glycohemoglobin with the Abbott IMx analyzer. Clin. Chem. 39; 2090-2097, 1993. 